\title{
Spontaneous Excitations in the Visual Cortex: Stripes, Spirals, Rings, and Collective Bursts
}

\author{
Corinna Fohlmeister \\ Wulfram Gerstner \\ Raphael Ritz \\ J. Leo van Hemmen \\ Physik-Department der TU München, \\ D-85747 Garching bei München, Germany
}

As a simple model of the cortical sheet, we study a locally connected net of spiking neurons. Refractoriness, noise, axonal delays, and the time course of excitatory and inhibitory postsynaptic potentials are taken into account explicitly. In addition to a low-activity state and depending on the synaptic efficacy, four different scenarios evolve spontaneously, viz., stripes, spirals, rings, and collective bursts. Our results can be related to experimental observations of drug-induced epilepsy and hallucinations.

\section{Introduction}

What do spontaneous coherent excitations in the primary visual cortex look like in time and-that is what we are interested in here-in space? This is a fascinating question whose solution is, to some extent, now within reach of computational neuroscience. It is generally believed that this kind of excitation occurs in drug-induced epilepsy and, presumably, also in hallucinations.

Hallucinations (Klüver 1967; Siegel and West 1975; Siegel 1977; Cowan 1985) are perceptions in the absence of a visual stimulus. They can occur even in subjects that have been completely blinded by a retinal disease (Zeki 1993). It was Klüver (1967) who in the twenties started experiments to classify what he called "form constants," which meanwhile have turned out to be universal characteristics of the first stage of druginduced imagery, most notably LSD. There are at least four categories of form constant, such as grating and filigree, spiral, tunnel and funnel, and cobweb. The imagery of the second stage is much more complex and, without any doubt, involves several areas of the brain. We mention two key questions: Are the form constants generated in the primary visual cortex (areas V1 and V2) or are they due to functional feedback, i.e., feedback from other areas with different functions? Second, can we understand the form constants theoretically? 
There exists a mathematically very elegant analysis of Ermentrout and Cowan (1979). Their main hypothesis, which we will adopt as well, is that the form constants can be modeled as elementary excitations in the primary visual cortex. The model uses a rate coding and takes the complex logarithm (Schwartz 1977) as the retinocortical map. The patterns follow from a bifurcation analysis in a neighborhood of the homogeneous low-activity state, a linear theory. A final result is that parallel stripes of active and quiescent neurons constitute elementary excitations of the model. Due to the retinocortical map, some of the cortical stripe patterns should appear as spirals on the retina. One may wonder, though, what are the spontaneous excitations in a "realistic" nonlinear cortical network of spiking, noisy neurons? This is the question we will focus on. In so doing we can, and will, verify the above hypothesis. In the context of our model we conclude that several, but not all, form constants occur as spontaneous excitations. Furthermore, we do encounter spatiotemporal activity patterns as found experimentally in drug-induced epilepsy.

At the same time as us but in a network of integrate-and-fire neurons without delays, local inhibition, and noise, Milton et al. (1993) found spirals as elementary excitations that evolve out of a fixed excitation center. Spirals are inconsistent with the parallel stripes referred to above. Below we will clear the situation and show that there is in fact a sequence of at least four scenarios. In so doing we will avoid any external input and exploit several neural characteristics which have been incorporated into our own spike response model.

\section{Spike Response Model}

The essentials of neuronal behavior are the absolute and relative refractory period, the response at the soma resulting from synaptic input (usually described by an alpha function), the omnipresent delays, and noise. All these ingredients have been incorporated in the spike response model (Gerstner and van Hemmen 1992, 1993; Gerstner et al. 1993). It presents a faithful but simplified description of the neurons themselves without taking recourse to differential equations. This is essential since we have to study the spatial activity of a large system of neurons (say $N \geq 20,000$ ) over a long period of time.

We discretize time by units $\Delta t=1 \mathrm{msec}$, the width of a spike, and label the neurons on a two-dimensional square lattice by the index $i$. The state of a neuron is described by $S_{i} \in\{0,1\}$. If the potential $h_{i}$ at the hillock of neuron $i$ reaches the threshold $y$, then the neuron is expected to fire. We describe this stochastic behavior through a noise parameter $\beta$ in the transition probability

$$
\operatorname{Prob}\left\{S_{i}(t+\Delta t)=1 \mid h_{i}(t)\right\}=\frac{1}{2}\left\{1+\tanh \left[\beta\left(h_{i}(t)-\vartheta\right)\right]\right\}
$$


This is the conditional probability that neuron $i$ fires at time $t+\Delta t$ given $h_{i}(t)$. In the noise-free limit $\beta \rightarrow \infty$ we get $S_{i}(t+\Delta t)=\Theta\left[h_{i}(t)-\vartheta\right]$ where $\Theta$ is the Heaviside step function: $\Theta(x)=1$ for $x \geq 0$ and $\Theta(x)=0$ for $x<0$. In the numerics to be described below we have taken $\beta=25$ and $\vartheta=0.12$.

The spike response model describes the response of a neuron-both the sender and the receiver-to a spike. If a neuron has fired a spike, it exhibits refractory behavior for a while, i.e., it cannot or can hardly spike. This is taken care of by the refractory function $\eta$, which is $-\infty$ during the absolute refractory period and negative but increasing to zero thereafter,

$$
h_{j}^{\mathrm{refr}}(t)=\sum_{\tau \geq 0} \eta(\tau) S_{j}(t-\tau)
$$

Here we take $\eta(\tau)=-\infty$ for $\tau=1$ and zero elsewhere.

The spike travels along an axon and reaches a synapse on the dendritic tree of neuron $i$ after $\Delta_{i}$ msec. Let the synaptic strength be $J_{i j}$ and denote the alpha function by $\varepsilon$. Then we obtain for the total input at the hillock of neuron $i$

$$
h_{i}^{\text {syn }}(t)=\sum_{j} J_{i j} \sum_{\tau \geq 0} \varepsilon(\tau) S_{j}\left(t-\tau-\Delta_{i}\right)
$$

where $\varepsilon(\tau)=\tau / \tau_{\varepsilon}^{2} \exp \left(-\tau / \tau_{\varepsilon}\right)$ so that $\sum_{\tau} \varepsilon(\tau)=1$; here $\tau_{\varepsilon}=2 \mathrm{msec}$. For the sake of computational simplicity we have assumed that the delays $\Delta_{i}$ depend on $i$ (instead of, say, $j$ ). In this work the $\Delta_{i}$ are taken from $\{0,1,2\}$ with equal probability. Furthermore, $J_{i i}$ always vanishes.

The neurons considered so far are pyramidal cells. The stellate cells are modeled by an inhibitory loop, which is assigned to each neuron,

$$
h_{i}^{\mathrm{inh}}(t)=\sum_{\tau \geq 0} \varepsilon^{\mathrm{inh}}(\tau) S_{i}\left(t-\tau-\Delta_{i}^{\mathrm{inh}}\right)
$$

where $\varepsilon^{\text {inh }}(\tau)$ first assumes a strongly negative value during $5 \mathrm{msec}$ (shunting inhibition) and then decays exponentially with a time constant $\tau_{\text {inh }}=6$ msec. Moreover, $\Delta_{i}^{\text {inh }} \in\{3,4,5\}$ is a uniformly distributed random variable. It is known that stellate cells operate locally. This we have simplified to a strictly local interaction; for details, see Gerstner et al. (1993). Putting things together we find

$$
h_{i}(t)=h_{i}^{\text {refr }}(t)+h_{i}^{\text {syn }}(t)+h_{i}^{\text {inh }}(t)
$$

which is to be substituted into 2.1 . What is left is specifying the $J_{i j}$ in 2.3 .

Since we are concerned with visual percepts such as hallucinations it seems natural, even imperative (Zeki 1993), to model the primary visual cortex. We will work with a simplified model of cortical connectivity. Inside a column the pyramidal cells experience an excitatory interaction. 
Different columns with strongly different direction preferences are expected to inhibit each other. The upshot is a "mexican hat,"1

$$
J_{i j}=A \exp \left(-r_{i j}^{2} / \lambda_{1}\right)-B \exp \left(-r_{i j}^{2} / \lambda_{2}\right)
$$

with $\lambda_{1} \ll \lambda_{2}$ and $A \gg B$. Here $r_{i j}$ is the Euclidean distance between $i$ and $j$. A second possibility, which has also been studied, is

$$
J_{i j}=\hat{A} \quad \text { for } r_{i j} \leq r_{0} \quad \text { and } \quad-\hat{B} \quad \text { for } r_{0}<r_{i j} \leq r_{\max }
$$

with $r_{\max } \leq 30$ and, again, $\hat{A} \gg \hat{B}$. We use free boundary conditions. In our numerical simulations we have seen no difference between 2.6 and 2.7. Alternatively and giving rise to the very same scenarios, one can replace $J_{i j}$ in 2.3 by $D \tilde{J}_{i j}$ where $\tilde{J}_{i j}=1$ with probability $\left.\exp \left[-\left(r_{i j}-1\right) / \lambda_{\exp }\right)\right]$ or $\left.\exp -\left[\left(r_{i j}-1\right) / \lambda_{\text {Gauss }}\right)\right]^{2}$; otherwise $\tilde{I}_{i j}$ vanishes. Typical values for the $\lambda s$ are in the range between 2 and 5 . The probabilities have been chosen in such a way that nearest neighbors $\left(r_{i j}=1\right)$ are always connected. $D$ is a drug parameter.

Summarizing, we have explicitly modeled the various interactions including the stellate cells, the delays that are abundantly present in the cortex, and the noise. We now turn to the network behavior itself.

\section{Drug-Induced Collective Excitations}

As in the experiments (Siegel and West 1975; Siegel 1977), we study a network without external input. In its normal state we then encounter spontaneous activity in the form of incoherent low-frequency firing. Fixing $B$ (or $\hat{B}$ ) and increasing the excitatory $A, \hat{A}$, or $D$, so as to model the influence of hallucinogens, we find four successive scenarios (see Figs. 1-4). We always start with random initial conditions, unless stated otherwise, and find depending on $A, \hat{A}$, or $D$ :

1. Stripes. Once $A$ (or $\hat{A}$, or $D$ ) has become large enough, say $A>A_{c}^{(0)}$, an excitation can propagate through the lattice. Just above $A_{c}^{(0)}$ the stripes are relatively short but they become longer with increasing $A$ (see Fig. 1). As time proceeds, the stripes propagate. Their length does not grow but they get slightly curved (the more so with increasing $A$ ) as the neurons in the center of a line segment are stimulated more strongly than those at the ends and, hence, their propagation is faster. Behind a stripe the neurons experience inhibition due to the stellate cells which get activated a bit later.

2. Spirals. As $A$ (or $D$ ) increases further, the stripes get longer and more curved so that for $A>A_{c}^{(1)}$ they regroup and build a spiral (see

\footnotetext{
${ }^{1}$ Interestingly, Hebbian learning of random contours gives rise to the very same form. It is plain that Dale's law is inconsistent with a Mexican hat but this form has been very popular. It is a simple matter, though, to redefine the sign of the bonds and at the same time shift the threshold $\vartheta$.
} 

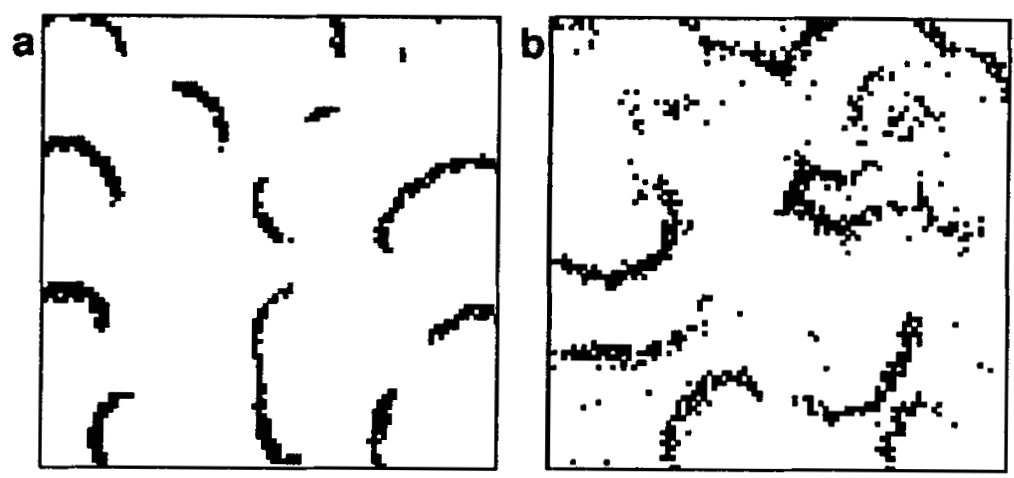

Figure 1: Scenario $1-$ stripes. (a) $90 \times 90$ network with locally homogeneous couplings $\hat{A}=0.16, \hat{B}=0.02$, while $r_{0}=15$ and $r_{\max }=20$; cf. 2.7 . (b) $90 \times 90$ network with locally sparse, excitatory couplings whose probability decreases with the distance; cf. Section 2 . Here $\lambda_{\text {Gauss }}=2$ and $D=0.056$. Note the similarity of the two figures despite their different microscopic structure. For all figures we have taken random initial conditions.
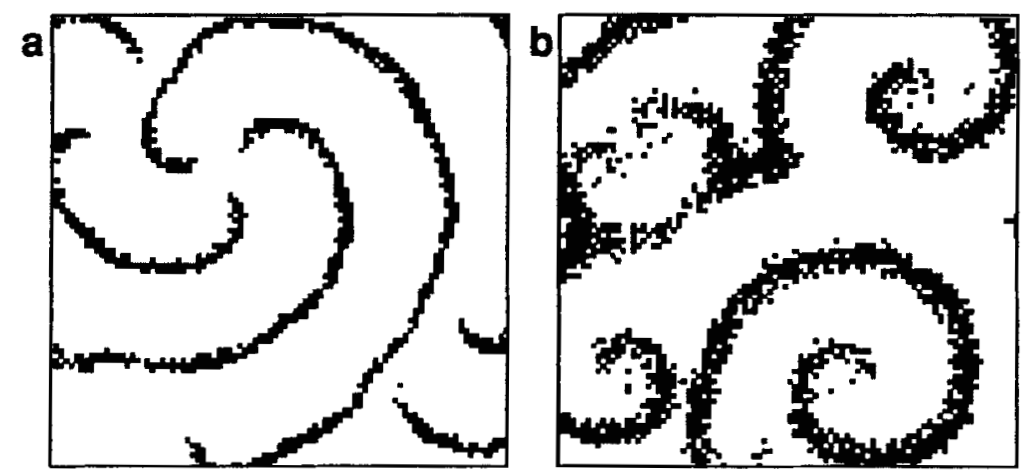

Figure 2: Scenario 2-spirals. (a) $90 \times 90$ network with $A=0.12, B=0.02$, $\lambda_{1}=15$ and $\lambda_{2}=100$; cf. 2.6 . Two or more spirals may coexist as shown in (b), where we have a $90 \times 90$ network with excitatory couplings whose probability decreases with the distance; cf. Section 2 . Here $\lambda_{\text {Gauss }}=2.83$ and $D=0.1$. 

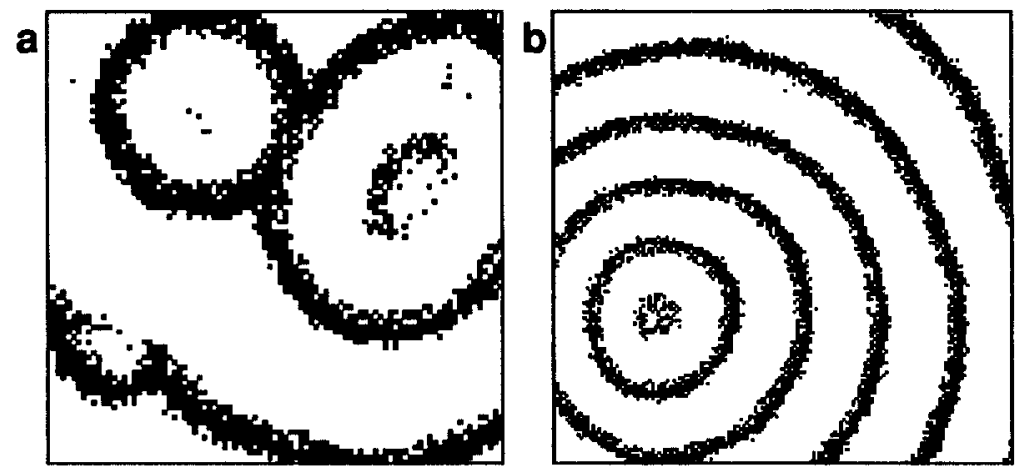

Figure 3: Scenario 3-rings. (a) $90 \times 90$ network with $A=0.14, B=0.02, \lambda_{1}=15$ and $\lambda_{2}=100$; cf. 2.6. The two rings annihilate each other where they meet. New rings originate from the two centers. In (b) we show a $150 \times 150$ network with excitatory couplings whose probability decreases with the distance; cf. Figures $1 \mathrm{~b}$ and $2 \mathrm{~b}$. Here $\lambda_{\text {Gauss }}=2.83$ and $D=0.12$.
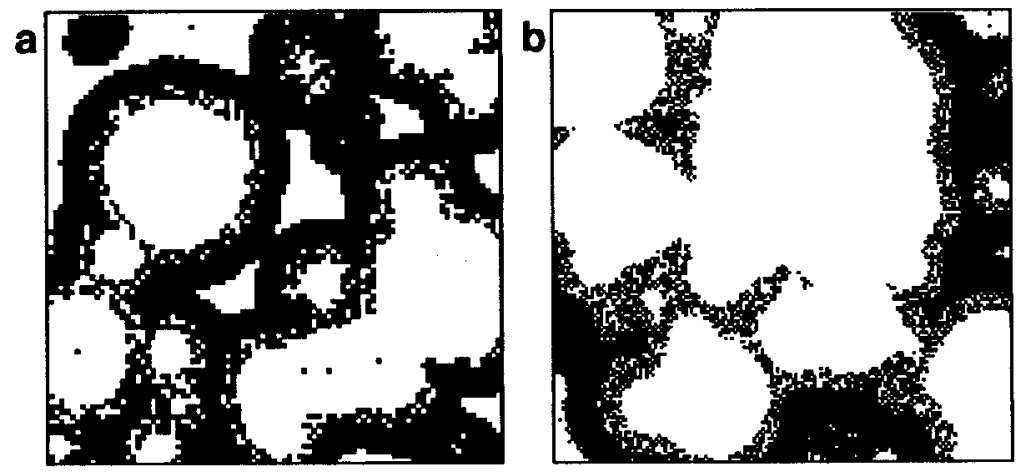

Figure 4: Scenario 4-collective burst. (a) $90 \times 90$ network with $A=2.4, B=0.02$, $\lambda_{1}=8.4$ and $\lambda_{2}=100$; cf. 2.6 . (b) $150 \times 150$ network with excitatory couplings whose probability decreases with the distance; cf. Figures $1 b-3 b$. Here we have an exponential distribution with $\lambda_{\exp }=3$. Furthermore, $D=0.14$. 
Fig. 2). Plainly, spirals rotate. The number of their arms (1, 2, or 3) depends on the random initialization. Spirals are also extremely stable. Once they exist one can even increase $A$ suddenly to a strength corresponding to scenario 4 but nevertheless the spirals survive.

3. Rings (see Fig. 3). There may be several centers generating new rings all the time. These propagate outward. If two nonconcentric rings hit each other, they annihilate their common part while moving outward. The reason is simply the inhibition that follows a front. The thickness of a ring increases with $A$ or $D$, respectively.

4. Collective bursts. These are complex pulsating patterns. Here $A$ (or $D$ ) is so large that a few active neurons ignite the whole system in 20-25 msec (cf. Fig. 4) after which inhibition takes over and a quiescent state sets in. The frequency is in a range between 10 and $20 \mathrm{~Hz}$. The resulting activity pattern vaguely resembles an epileptic state.

Interestingly, and in agreement with experiments (Siegel 1977), the "objects" in scenarios 2 and 3 have different length scales that vary from one scene to the next (even for the academic case of fixed parameter values). The width of the stripes in scenario 1 depends on $A$ (or $D$ ). The patterns in scenario 4 have all length scales. Indirect experimental evidence confirming scenarios 2-3 has been found by Petsche et al. (1974) in the occipital cortex of a rabbit with penicillin-induced epilepsy. Quite surprisingly, even for the complex pattern of scenario 4 experimental data are available (cf. Siegel and West 1975, p. 123).

\section{Discussion}

The "wetware" of the primary visual cortex apparently allows a variety of spontaneous excitations that are similar to patterns found in excitable media (Tyson and Keener 1988; Meron 1992; Cross and Hohenberg 1993). They arise due to intrinsic nonlinearities of the neuronal dynamics and resemble experimental hallucinogen-induced activity patterns rather closely but not completely. These excitations, however, are in the cortex and it is a natural question what they would look like on the retina. To answer this question we have taken Figure $2 a$, positioned it extrafoveally so that the complex logarithm offers a fair description of the retinocortical map, and applied the inverse map. The result is shown in Figure 5 where the Archimedean spiral in the cortex reappears as a quasi-logarithmic spiral on the retina. In passing we note that we find several but not all four types of "form constant" as described by Klüver (1967). This may be due to the initial conditions that we had chosen, viz., random ones. It is an open problem, though, what are the generic initial conditions that generate, e.g., a hexagonal pattern.

The performance of a large network does not depend on the details of the model once the neural essentials have been incorporated. An example is provided by the three different kinds of coupling that we assumed 

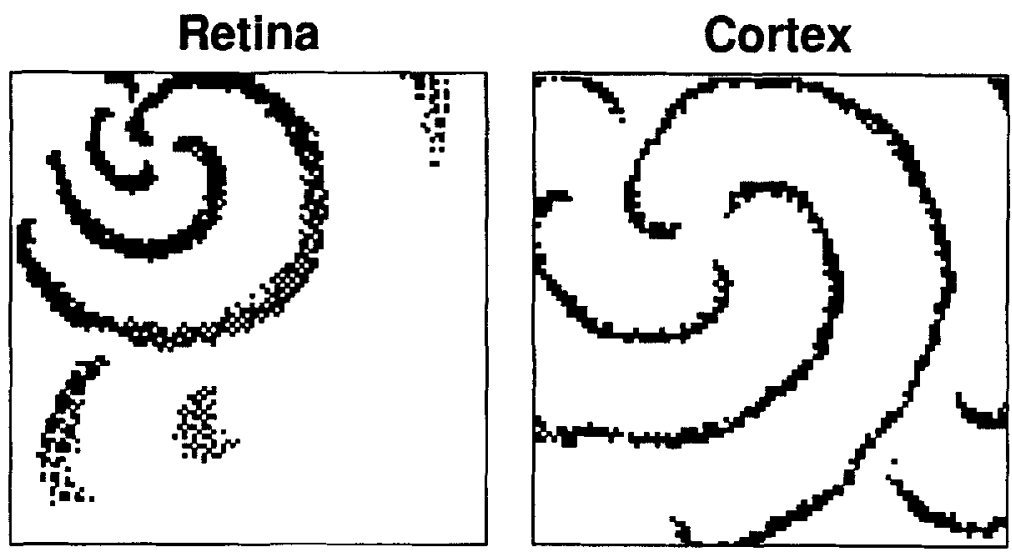

Figure 5: Retinal pattern (left) corresponding to the cortical activity pattern of Figure $2 \mathrm{a}$ (right). The retinal picture is the result of the inverse retinocortical map applied extrafoveally; cf. Schwartz (1977). If $(x, y)$ is a point in the cortex and $(r, \varphi)$ is on the retina, then parameters have been chosen in such a way that $r=\exp x$ and $\varphi=y$.

in the primary visual cortex, viz., the locally homogeneous ones (2.6) and (2.7) and the locally sparse, excitatory ones whose probability decreases with the distance (cf. Figs. 1-4). It has been stressed by Zeki (1993, pp. 324-326 and 342-343) that hallucinations do depend on reentry into area V1 or V2. On the basis of the present work and in agreement with Ermentrout and Cowan (1979) we tentatively suggest that the form constants are mainly generated in the primary visual cortex. Through functional feedback they may be, and we expect are, modified and combined with other objects, e.g., from memory. Under this proviso we are then led to the following interpretation. The scenarios 2 and 3 are in a one-to-one correspondence with the experimental hallucinatory spirals, tunnels and funnels-the more so since spirals are very stable and, thus, dominant. They also have been observed indirectly in drug-induced epilepsy. On the other hand, scenario 1 gives room to many interpretations. Scenario 4 is a high-dose one and hard to reach since the system usually has to pass through the previous three scenarios, where it can get stuck. Nevertheless, it has been "seen." One has to realize, though, that pictures drawn by patients may give rise to contradictory results as is illustrated nicely by Siegel and West (1975, p. 135). Here both a quasi-logarithmic spiral, which is "seen" by most people, and a purely Archimedean one are shown; the two spirals were observed by two different persons under 
the influence of ketamine and LSD, respectively. In fact, the two different pictures with Archimedean and logarithmic spirals would constitute a fascinating problem to theory, if they were reproducible.

In summary, we have exhibited several scenarios that appear as the synaptic efficacy is increased in a locally connected neuronal network. All of them have been observed, some in the cortex, others through hallucinations. Our model reproduces some but not all of the form constants as they are found in hallucinations. Hence it may well be that the basic hypothesis that they are generated in the primary visual cortex is too simple-minded in relation to cortical processing. There is little doubt, however, that all these spontaneous excitations with their typical spatiotemporal behavior do occur in the cortex.

An analytic treatment of the model under consideration will be presented elsewhere (Fohlmeister et al. 1995).

\section{Acknowledgments}

WG has been supported by the Deutsche Forschungsgemeinschaft under Grant He 1729/2-2.

\section{References}

Cowan, J. D. 1985. What do drug-induced visual hallucinations tell us about the brain? In Synaptic Modification, Neuron Selectivity, and Nervous System Organization, W. B. Levy, J. A. Anderson, and S. Lehmkuhle, eds., pp. 223241. Lawrence Erlbaum, Hillsdale, NJ.

Cross, M. C., and Hohenberg, P. C. 1993. Pattern formation outside of equilibrium. Rev. Mod. Phys, 65, 851-1112.

Ermentrout, G. B., and Cowan, J. D. 1979. A mathematical theory of visual hallucination patterns. Biol. Cybern. 34, 137-150.

Fohlmeister, C., Gerstner, W., Ritz, R., and van Hemmen, J. L. 1995. Manuscript in preparation.

Gerstner, W., and van Hemmen, J. L. 1992. Associative memory in a network of 'spiking' neurons. Network 3, 139-164.

Gerstner, W., and van Hemmen, J. L. 1993. Coherence and incoherence in a globally coupled ensemble of pulse-emitting units. Phys. Rev. Lett. 71, 312315.

Gerstner, W., Ritz, R., and van Hemmen, J. L. 1993. A biologically motivated and analytically soluble model of collective oscillations in the cortex: I. Theory of weak locking. Biol. Cybern. 68, 363-374.

Klüver, H. 1967. Mescal and the Mechanisms of Hallucination. The University of Chicago Press, Chicago.

Meron, E. 1992. Pattern formation in excitable media. Phys. Rep. 218, 1-66.

Milton, J. G., Chu, P. H., and Cowan, J. D. 1993. Spiral waves in integrate-andfire neural networks. In Neural Information Processing Systems, S. J. Hanson, 
J. D. Cowan, and C. L. Giles, eds., Vol. 5, pp. 1001-1006. Morgan Kaufmann, San Mateo, CA.

Petsche, H., Prohaska, O., Rappelsberger, P., Vollmer, R., and Kaiser, A. 1974. Cortical seizure patterns in multidimensional view: The information content of equipotential maps. Epilepsia 15, 439-463.

Schwartz, E. 1977. Spatial mapping in the primate sensory projection: Analytic structure and relevance to perception. Biol. Cybern. 25, 181-194.

Siegel, R. K. 1977. Hallucinations. Sci. Am. 237(4), 132-140.

Siegel, R. K., and West, L. J. 1975. Hallucinations: Behavior, Experience, and Theory. Wiley, New York.

Tyson, J. J., and Keener, J. P. 1988. Singular perturbation theory of travelling waves in excitable media (a review). Physica D 32, 327-361.

Zeki, S. 1993. A Vision of the Brain. Blackwell Scientific, Oxford.

Received April 11, 1994; accepted December 22, 1994. 